\title{
P-0673 Correlation of serum adiponectin and NGAL levels in patients with type 1 diabetes and diabetic nephropathy
}

\author{
T. Mokhort ${ }^{1}$, A. Sazonava ${ }^{1,2}$, V. Shyshko ${ }^{1}$, A. Mokhort ${ }^{1}$, N. Karlovich ${ }^{1}$ \\ Belarusian State Medical University, Minsk, Belarus ${ }^{1}$ \\ City Endocrinological Dispensary, Minsk, Belarus²
}

\section{adipocyte}

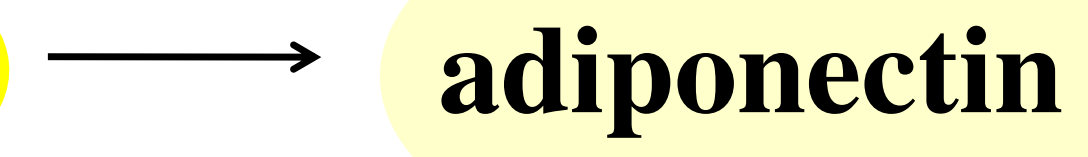

Thyroid function

Insulin

Glucagon

Inflammation

Angiotensin II

GLP-1

Vitamin D

Glucocorticoids

\begin{abstract}
Aims. Diabetic nephropathy (DN) is a leading cause of chronic kidney disease (CKD) and is responsible for approximately 30$40 \%$ of all end-stage renal disease (ESRD) cases. Early intervention in DN can improve prognosis but requires more accurate detection by using novel biomarkers from the early beginning of renal failure. Neutropfil gelatinase-associated lipocalin (NGAL) was shown to be highly useful alongside serum creatinine and other biomarkers in assessing kidney injury. Renal dysfunction may affect not only glycemic control, but also metabolism of biologically active cytokines such as adiponectin. Taking into account multiple effects of adiponectin the purpose of the study was to investigate potential relationship between serum adiponectin levels and diabetes compensation, BMI, NGAL levels and GFR in patients with type 1 diabetes (T1D) and comorbid CKD.
\end{abstract}

Materials and methods. We recruited 69 patients (21 male; 48 female; age 41,33 $\pm 11,491$ years; BMI 25,06 $\pm 4,722 \mathrm{~kg} / \mathrm{m} 2$; duration of T1D 22,11 $\pm 9,049$ years) at CKD stages 1, 2, 3, 4 $(\mathrm{N}=21 ; 30 ; 16 ; 2$, respectively). Hypertension was observed in $48(69,57 \%)$ patients, $38(55,07 \%)$ patients took ACE inhibitors. Changes in lipid profile were found in $55(79,71 \%)$ patients, and only $15(21,74 \%)$ of them received statins. GFR was estimated by CKD-EPI formula. All patients were divided into 2 groups according to GFR. The group 1 comprised 51 patients with GFR $>60 \mathrm{~mL} / \mathrm{min}$, group 2 - 18 patients with GFR $<60 \mathrm{~mL} / \mathrm{min}$. Biochemical parameters, lipid profile, HbA1c level, NGAL, serum adiponectin levels were measured. Nonparametric statistical methods were used. A P-value <0,05 was considered significant.
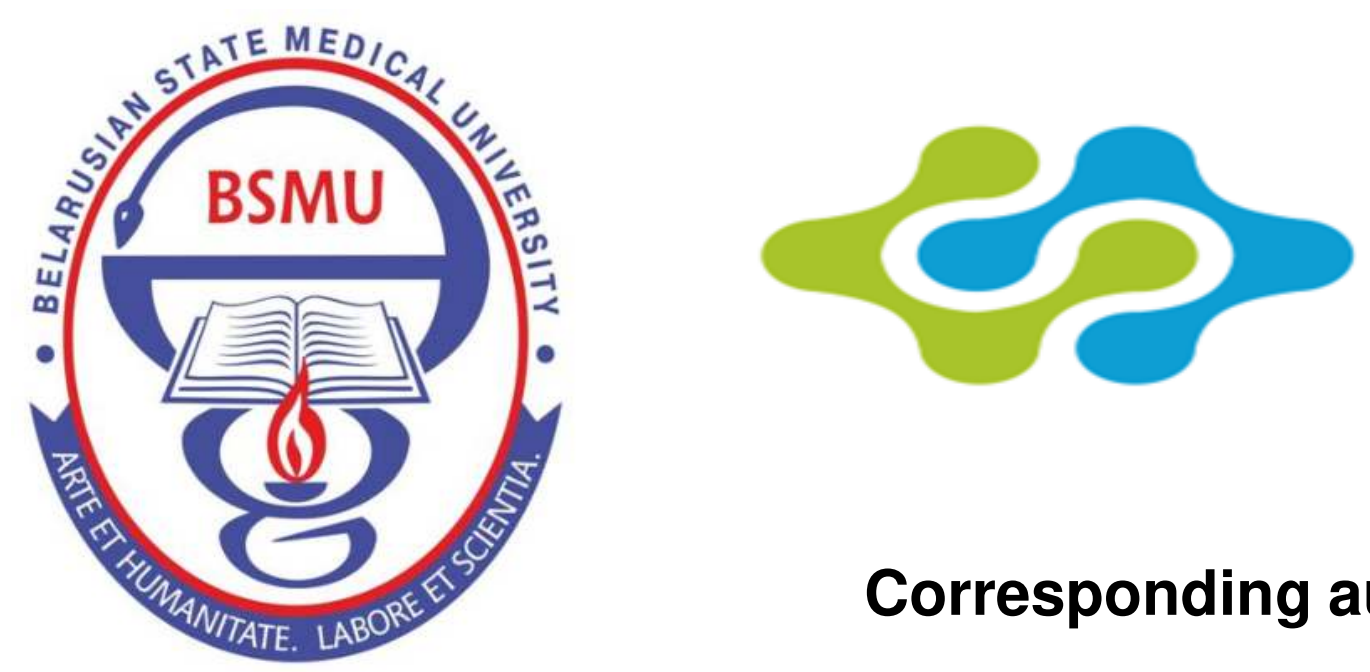

Results. The groups were matched by age, gender, BMI, duration of T1D, HbA1c levels. In patients with GFR $>60 \mathrm{~mL} / \mathrm{min}$ statistically significant inverse correlation between NGAL and adiponectin levels was observed ( $\mathrm{r}=-$ 0,534). Similar interdependence in patients with more severe GFR decrease was not obtained. The increase in adiponectin levels is most likely due to the non-absolute content of adiponectin in the serum, but the relative increase in the amount due to the decrease in renal clearance.

$\begin{array}{lccc} & \begin{array}{l}\text { GFR }>60 \\ \mathbf{m L} / \mathbf{m i n} \\ \mathbf{N}=\mathbf{5 1}\end{array} & \begin{array}{c}\text { GFR<60 } \\ \mathbf{m L} / \mathbf{m i n} \\ \mathbf{N}=\mathbf{1 8}\end{array} & { }^{*} \mathrm{p}<\mathbf{0 , 0 5} \\ & 13,64[9,25 ; & 20,73[12,46 ; & \mathrm{p}=0,020 \\ \text { Adiponectin, } & 16,61] & 28,48] & \\ \mathbf{m k g} / \mathbf{m l} & 0,88[0,48 ; & 1,98[0,91 ; & \mathrm{p}=0,003 \\ \mathbf{N G A L}, \mathbf{n g} / \mathbf{m l} & 1,30] & 3,29] & \\ & & & \end{array}$

No relationship was found between adiponectin and BMI, GFR and HbA1c. In both groups NGAL and adiponectin levels did not correlate with albumin/creatinine ration, duration of renal impairment, duration of T1D. In patients with GFR>60 mL/min NGAL correlates with HDL-c $(r=-0,20)$. The same correlation was observed in adiponectin $(\mathrm{r}=0,492)$, that proves its antiatherosclerotic effect. Adiponectin levels were inversely proportional to insulin units per day $(\mathrm{r}=-0,410)$. In patients with GFR $<60 \mathrm{~mL} / \mathrm{min}$ adiponectin inversely correlates with serum protein and albumin levels $(r=-0,749$ and $r=-0,729$, respectively).

Conclusion. In patients with T1D and diabetic nephropathy decline of GFR which leads to increase in serum NGAL levels is accompanied by an increase of serum adiponectin levels. Despite relative hyperadiponectinemia in CKD stages 3-4, protective metabolic effects of adiponectin are lost and this occurs independently of GFR and diabetes compensation, but mainly due to mechanisms involved in progression of renal damage and developing of systemic inflammation. 